\title{
Indemnisation du chômage : la réforme est en marche
}

\section{Maryse Badel}

\section{Q OpenEdition}

1 Journals

\section{Édition électronique}

URL : https://journals.openedition.org/rdctss/2054

DOI : $10.4000 /$ rdctss. 2054

ISSN : 2262-9815

\section{Éditeur}

Centre de droit comparé du travail et de la sécurité sociale

\section{Édition imprimée}

Date de publication : 1 avril 2018

Pagination : 160-163

ISSN : 2117-4350

\section{Référence électronique}

Maryse Badel, «Indemnisation du chômage : la réforme est en marche », Revue de droit comparé du travail et de la sécurité sociale [En ligne], 1 | 2018, mis en ligne le 01 novembre 2021, consulté le 12 novembre 2021. URL : http://journals.openedition.org/rdctss/2054 ; DOI : https://doi.org/10.4000/ rdctss. 2054

\section{(c) (i) $\odot$}

Revue de droit comparé du travail et de la sécurité sociale est mise à disposition selon les termes de la Licence Creative Commons Attribution - Pas d'Utilisation Commerciale - Pas de Modification 4.0 International. 


\section{MARYSE BADEL}

UNIVERSITÉ DE BORDEAUX

\section{INDEMNISATION DU CHÔMAGE : LA RÉFORME EST EN MARCHE}

La couverture du chômage en France est doublement originale. Alors que le chômage peut être perçu comme un risque social par excellence et qu'il est l'un des neuf risques de sécurité sociale admis par la Convention n 102 de l'OIT, sa couverture est organisée en France en marge de la Sécurité sociale. Même si elle emprunte aujourd'hui aussi bien aux techniques de sécurité sociale avec les prestations qui relèvent de l'assurance et aux techniques de l'aide sociale avec les prestations qui relèvent de l'assistance, l'indemnisation du chômage est en effet gérée par des organismes spécifiques distincts des caisses de Sécurité sociale, Pôle emploi au niveau local pour le versement des prestations et l'Union nationale pour l'emploi dans l'industrie et le commerce (UNEDIC) pour la gestion nationale du Régime d'assurance chômage. Ceci s'explique par le contexte dans lequel a été créée la Sécurité sociale en 1945. Le marché du travail était alors marqué par une pénurie de maind'œuvre, ce qui n'était guère propice à placer le chômage au cœur des questions sociales, et les libéraux, par crainte que l'indemnisation ne favorise le développement d'un chômage volontaire, étaient hostiles à l'indemnisation systématique à laquelle aurait donné lieu l'instauration d'une assurance chômage ${ }^{1}$. Aussi, l'indemnisation du chômage fut instaurée en 1958, soit plus de 10 ans après la création de la Sécurité sociale. Elle intervint dans un contexte marqué par le renouvellement politique, par les perspectives de mutations industrielles et d'intégration de la France dans le marché commun, et par la volonté de favoriser la mobilité de la main-d'œuvre et la fluidité du marché du travail2 ${ }^{2}$ L'instauration de l'assurance chômage trouve ainsi son origine dans la négociation collective, contrairement à la Sécurité sociale et à l'aide sociale où la création des prestations et de leur régime juridique revient au législateur.

Ainsi, et c'est là sa seconde originalité, l'organisation de l'assurance chômage résulte aujourd'hui d'une articulation étroite de la loi et de la négociation collective, ce qui confère aux partenaires sociaux une place tout à fait particulière ${ }^{3}$. La loi détermine les grandes lignes du régime d'assurance chômage. L'article L5421-2 du Code du travail prévoit que ce régime distribue une allocation de base calculée, dans certaines limites, en fonction du salaire perçu, selon des conditions d'âge et pour une certaine durée. Les mesures d'application des dispositions légales font l'objet d'accords négociés et conclus entre les organisations représentatives d'employeurs et de salariés, organisations représentatives au

1 C. Daniel, C. Tuchzirer, L'État face aux chômeurs. L'indemnisation du chômage de 1884 à nos jours, Flammarion, 1999 C. Willmann, L'identité juridique du chômeur, éd. LGDJ, coll. Thèses, t. 32, 1998.

2 B. Oudin, "La convention du 31 décembre 1958 relative à la création de l'allocation-chômage », Droit social. 1959. 364.

3 J.-J. Dupeyroux, M. Borgetto, R. Lafore, Droit de la sécurité sociale, éd. Dalloz, coll. Précis, 18 e éd., $2015, n^{\circ} 1609 \mathrm{~s}$. 
niveau national et interprofessionnel ${ }^{4}$. L'accord est conclu pour une durée déterminée, deux ou trois ans en général, et il est agréé, après avis de l'autorité administrative, par le ministre chargé de l'emploi si cet accord est conforme à la législation en vigueur. Cet agrément rend la convention d'assurance chômage obligatoire et applicable à tous les employeurs et les salariés compris dans le champ territorial de l'accord. En l'absence d'accord ou d'agrément de celui-ci, les mesures d'application sont déterminées par décret en Conseil d'État. Le régime de solidarité relève en revanche des seuls pouvoirs publics, les partenaires sociaux n'étant pas compétents pour les prestations destinées à garantir un revenu minimal aux chômeurs qui ont épuisé leurs droits au régime d'assurance et aux diverses catégories de demandeurs d'emploi qui n'ont pas vocation à en relever ${ }^{5}$.

L'assurance chômage n'a pas échappé au processus réformateur du modèle social français engagé par le Président Macron élu au printemps 2017. Après les ordonnances visant à réformer le Code du travail, après la loi de financement de la Sécurité sociale pour 2018 qui a introduit des changements majeurs pour le financement de la couverture santé, largement fiscalisée, et pour l'organisation de la Sécurité sociale, la gestion de la Sécurité sociale des travailleurs indépendants étant désormais dévolue aux organismes du Régime général, de nouvelles réformes sont attendues, en particulier pour la formation professionnelle et pour l'apprentissage. Et l'assurance chômage, pourtant modifiée il y a moins d'un an ${ }^{6}$, vient de faire l'objet d'une nouvelle réforme, Emmanuel Macron souhaitant instaurer un nouveau régime de "chômage universel » qui indemniserait les indépendants, les artisans et les commerçants, et les salariés démissionnaires.

Dans ce contexte, les partenaires sociaux sont parvenus à un accord de principe le 22 février $2018^{7}$.Conformémentauxsouhaitsprésidentiels,l'accordnationalinterprofessionnel (ANI) de 13 pages prévoit d'ouvrir un droit aux allocations aux salariés démissionnaires. Ceci est largement nouveau car, jusqu'à présent, la démission privait en principe du droit à la prestation d'assurance chômage nommée allocation de retour à l'emploi (ARE). Ce n'était que de façon exceptionnelle que le salarié démissionnaire pouvait conserver ce droit, si sa démission était considérée comme légitime par l'organisme chargé du paiement et du placement des demandeurs d'emploi (Pôle emploi). Tel était le cas quand le salarié démissionnait pour suivre la personne avec laquelle il vivait en couple, après un mariage ou un pacte civil de solidarité (PACS), ou pour suivre ses parents s'il était mineur. Tel était encorele cas sile déménagement du salarié, motivéparle placement deson enfanthandicapé dans une structure éloignée ou par la nécessité de se soustraire à des violences conjugales, lui imposait de quitter son emploi. L'ANI de 2018 élargit considérablement le cercle des exceptions. Désormais, les salariés démissionnaires quittant leur emploi pour mener un nouveau projet professionnel pourront prétendre au bénéfice de l'assurance chômage dès lors que ce projet, après avoir été instruit par un conseil en évolution professionnelle (CEP), aura été validé par une instance indépendante de Pôle emploi. Ce projet devra être réel et sérieux, caractère qui sera apprécié au regard des besoins du marché du travail dans le ou les bassins d'emploi identifié(s). II devra aussi consister dans une formation qualifiante longue ou dans une formation complémentaire identifiée à la suite d'une validation des

4 C. trav., art. L5422-20 s.

5 C. trav., art. L5423-1 s.

6 Convention du 14 avril 2017 relative à l'assurance chômage, Protocole d'accord du 28 mars 2017 relatif à l'assurance chômage.

7 Accord national interprofessionnel du 22 février 2018 relatif à la réforme de l'assurance chômage. 
acquis de l'expérience. Le bénéfice de "l'allocation d'aide au retour à l'emploi projet » (AREP), équivalent de l'ARE, sera aussi soumis à une condition minimale de 7 ans dans l'emploi. Ce serait ainsi entre 14000 et 23000 salariés qui pourraient être concernés, et le coût du dispositif pour l'assurance chômage en année pleine serait compris entre 180 et 330 millions d'euros. L'objet de ce nouveau droit est, selon les partenaires sociaux, de sécuriser le parcours des salariés sur le marché du travail, d'accompagner la décision de démission et la préparation du projet d'évolution professionnelle pour que les risques de surestimation de retour emploi et de désinsertion professionnelle soient évités.

La question du chômage des indépendants qui avait fait l'objet d'un volumineux rapport au mois d'octobre $2017^{8}$ est aussi envisagée mais elle ne donne lieu qu'à des propositions a minima: le patronat étant hostile à de nouvelles cotisations patronales, I'ANI envisage la création d'un régime public de protection financé par l'impôt, ouvert à tous les travailleurs indépendants et distinct de l'assurance chômage des salariés, qui pourrait verser une prestation spécifique en cas de fait générateur strict comme la liquidation judiciaire de l'entreprise. Il envisage aussi que, dans la continuité de ce qui existe aujourd'hui, des régimes privés puissent proposer des prestations complémentaires mieux adaptées aux situations individuelles. Ce qui aurait été une transformation majeure du régime d'assurance chômage et qui aurait conduit, du même coup, à une véritable mutation du concept de chômage, ne s'est donc pas encore produit. Quant à la situation des travailleurs économiquement dépendants tels ceux des plateformes (Uber, Deliveroo, Airbnb...), elle ne fait l'objet d'aucune proposition, un groupe de travail étant chargé de rechercher des dispositifs appropriés les concernant et de rendre des conclusions avant la fin de l'année 2018.

Dans son article 3 intitulé "Dispositions relatives à l'emploi durable », l'ANI aborde la question épineuse des contrats courts d'1 mois ou moins. II relève que le nombre de ces contrats a presque triplé au cours de ces 20 dernières années et constate que deux-tiers d'entre eux correspondent à des relations de travail durables entre un même salarié et un même employeur. Afin d'éviter les logiques d'optimisation tant de la part des employeurs que des salariés et dans la continuité de l'accord d'avril 2017, l'ANI énonce que les organisations représentatives d'employeurs et de salariés dans l'ensemble des branches devront ouvrir des négociations pour déterminer les moyens de développer l'installation durable dans l'emploi et éviter les risques d'enferment dans des situations de précarité. Il programme leur déroulement. Les organisations de salariés et d'employeurs devront établir un diagnostic quantitatif et qualitatif des situations de recours aux contrats courts sous toutes leurs formes, des motifs de recours, et du niveau de qualification des salariés concernés par ces contrats et de leurs trajectoires professionnelles. Sur la base de ce diagnostic, les organisations représentatives devront adopter des mesures permettant de modérer leur utilisation, d'allonger les durées d'emploi, et prendre des mesures relatives à l'organisation du travail et à la gestion de l'emploi. Elles pourront encore décider de moyens pour accompagner le développement des compétences des salariés et pour favoriser l'accès à l'emploi durable des populations les plus éloignées de l'emploi. L'accord ou le relevé de conclusions résultant des négociations ouvertes est attendu avant le 31 décembre 2018, un bilan d'étape devant être réalisé avant le 31 juillet 2018. L'accord rappelle aussi que si ces mesures étaient insuffisantes, le gouvernement pourrait mettre en œuvre un système de bonus-malus sur les cotisations patronales d'assurance chômage.

8 IGAS IGF, « Ouverture de l'assurance chômage aux travailleurs indépendants », oct. 2017. 


\section{FRANCE}

Concernant la gouvernance et le pilotage de I'UNEDIC enfin, l'article 4 de l'ANI réaffirme que la négociation paritaire continue de "définir en toute autonomie les règles d'indemnisation et le niveau de ressources nécessaires" du régime, avec l'objectif, à terme, de se passer de la garantie financière accordée par l'État. Les partenaires sociaux demandent une "clarification» des responsabilités entre la solidarité nationale et l'assurance-chômage. Ils proposent ainsi la tenue d'une "conférence pour l'emploi » avec l'État afin de fixer des « objectifs communs au régime de solidarité », c'est-à-dire les minima sociaux, "et au régime assurantiel », c'est-à-dire l'assurance-chômage, et de réaffirmer les "principes fondamentaux d'un paritarisme utile».

L'ANI a été assez vivement critiqué par la ministre du travail Muriel Pénicaud qui a exprimé sa déception. Elle a regretté le caractère timoré des dispositions et a estimé que le «big-bang... nécessaire pour le système » n'était pas intervenu. La réforme doit être poursuivie dans un grand projet de loi qui sera présenté au Parlement. 